UCRL-JC-129025

PREPRINT

\title{
Propagation of Realistic Beams In Underdense Plasma
}

D. E. Hinkel, E. A. Williams, R. L. Berger,

L. V. Powers, A. B. Langdon, C. H. Still

This paper was prepared for submittal to the 39th Annual Meeting of the APS Division of Plasma Physics

Pittsburgh, PA

November 17-21, 1997

November 10, 1997

This is a preprint of a paper intended for publication in a journal or proceedings. Since changes may be made before publication, this preprint is made available with the understanding that it will not be cited or reproduced without the permission of the author. 


\section{DISCLAIMER}

This document was prepared as an account of work sponsored by an agency of the United States Government. Neither the United States Government nor the University of California nor any of their employees, makes any warranty, express or implied, or assumes any legal liability or responsibility for the accuracy, completeness, or usefulness of any information, apparatus, product, or process

disclosed, or represents that its use would not infringe privately owned rights. Reference herein to any specific commercial product, process, or service by trade name, trademark, manufacturer, or otherwise, does not necessarily constitute or imply its endorsement, recommendation, or favoring by the United States Government or the University of California. The views and opinions of authors expressed herein do not necessarily state or reflect those of the United States Government or the University of California, and shall not be used for advertising or product endorsement purposes. 


\title{
Propagation of realistic beams in underdense plasma
}

\author{
D. E. Hinkel, E. A. Williams, R. L. Berger, L. V. Powers, A. B. Langdon, and C. H. Still \\ Lawrence Livermore National Laboratory \\ P.O. Box 808, Livermore, CA 94550
}

(November 10, 1997)

\begin{abstract}
The effect of beam structure on propagation through underdense plasma is examined in two different examples. First, it is shown that the distribution of intensities within a laser beam affects how the beam deflects in the presence of transverse plasma flow. A detailed analysis of beam deflection shows that the rate scales linearly with intensity and plasma density, and inversely with plasma temperature. When the plasma flow is subsonic, the deflection rate is proportional to the ion damping decrement, and scales as $M /\left(1-M^{2}\right)^{3 / 2}$, where $M$ is the transverse flow Mach number. When the plasma flow is supersonic, the deflection rate scales as $\left.1 /\left[M\left(M^{2}-1\right)\right)^{1 / 2}\right]$. Next, the effect of beam structure on channel formation by very intense laser beams is studied. A diffraction-limited beam with $40 \mathrm{TW}$ of input power forms a channel through $400 \mu \mathrm{m}$ of plasma, whereas when this beam is phase aberrated, channel formation does not occur.

52.40.Db,52.35.Mw,52.40.Nk,52.60.th
\end{abstract}

Typeset using REVTEX 


\section{INTRODUCTION}

The study of parametric instabilities [1] has long been a topic of interest in laser-plasma interactions. Reliable prediction of experimental results requires detailed knowledge of the plasma characteristics (density, temperature and flow profiles) as well as the laser beam structure (phase and intensity modulation information). Hydrocodes such as Lasnex [2] as well as experimental measurements yield data about the plasma, and experimental measurements [3-5] and laser beam propagation codes [6] provide relevant information about beam characteristics.

In this article we examine two physical mechanisms which demonstrate the effect of beam structure on its propagation through underdense plasma. First, we discuss the effect of transverse plasma flow on beam deflection. When there is plasma flowing across a beam, the density depressions created by the ponderomotive pressure of the light wave are swept downstream in the flow direction, and the light, which is refracted into the density depression, moves downstream as well. We present a simple analysis of this problem, which shows that the beam deflection rate scales with the figure of merit $Q \equiv\left(v_{0} / v_{e}\right)^{2}\left(n / n_{c}\right)\left(1-n / n_{c}\right)^{-1}$, where $v_{0}\left(v_{e}\right)$ is the electron quiver (thermal) velocity, $n$ is the electron plasma density, and $n_{c}$ is the critical plasma density at which light reflects $\left(n / n_{c}=\omega_{p e}^{2} / \omega_{0}^{2}\right.$, where $\omega_{p e}$ is the electron plasma frequency, and $\omega_{0}$ is the light wave frequency). We have derived an expression for the beam deflection that is valid for subsonic transverse flow $(M \equiv u / C,<1$, where $u$ is the transverse plasma flow and $C_{\text {. }}$ is the sound speed), near-sonic flow $(M \approx 1)$, and supersonic flow $(M>1)$. In the subsonic regime, the beam deflection rate scales as $\bar{\nu} M /\left(1-M^{2}\right)^{3 / 2}$, where $\bar{\nu}=$ constant is the ion damping decrement. For $M \approx 1$, this scaling becomes $\bar{\nu}^{-1 / 2}$, and for $M>1$, the scaling is $1 /\left[M\left(M^{2}-1\right)^{1 / 2}\right]$, independent of the ion damping decrement. This scaling is in agreement with the results of Ghosal and Rose [7].

Recent experiments in gas-filled hohlraums conducted on the Nova laser at Lawrence Livermore National Laboratory (LLNL) show the laser spot on the hohlraum wall is $150 \mu \mathrm{m}$ closer to the laser entrance hole (LEH) than in empty hohlraum experiments [3]. We show 
that this spot motion can be interpreted as beam deflection that occurs near the LEH by simulating these experiments using F3D [8], a fully nonlinear, three-dimensional (3D) fluid hydrodynamics code with paraxial light wave propagation. A necessary component in effectively modelling the experiment is utilizing as input to F3D the beam structure of the unsmoothed Nova laser beams [6] as well as realistic plasma profiles as generated by Lasnex. Our modelling shows that an unsmoothed Nova beam undergoes beam deflection at the LEH which results in a $110 \mu \mathrm{m}$ shift in the beam centroid toward the LEH. When the beam is spatially smoothed by applying random phase plates (RPPs) [10], the centroid shift is reduced to $40 \mu \mathrm{m}$. This is in quantitative agreement with experiments.

The second physical mechanism we address in this article is the effect of beam structure on channel formation by ultra-intense laser light, a situation directly applicable to the holeboring beam in the fast ignitor concept [11]. We have modified F3D to include relativistic corrections and the effect of charge separation [17]. In 3D simulations of a Gaussian beam with peak intensity $5.7 \times 10^{17} \mathrm{~W} / \mathrm{cm}^{2}$ and a waist of $15 \mu \mathrm{m}$, or of an $\mathrm{f} / 3$ beam with the same amount of input power as the Gaussian, the beam creates a channel through $1 \mathrm{~mm}$ of plasma with initially uniform density $n=0.1 n_{c}$ for the Gaussian beam or through 0.4 $\mathrm{mm}$ for the $\mathrm{f} / 3$ beam in a time $t=15 \mathrm{ps}$. However, when random phase aberrations are superposed on an $f / 3$ laser beam, so that asymptotically the beam is $f / 3$, but at best focus is no longer diffraction limited [its spot size is $15 \mu \mathrm{m}$ ], a channel does not form through $400 \mu \mathrm{m}$ of plasma at density $n=0.1 n_{c}$ in a time $t=30 \mathrm{ps}$.

The outline of this paper is as follows. Section II addresses beam deflection. In Section IIA, we present our analysis, and in Section IIB the results of our modelling. Section III is devoted to channel formation of ultra-intense laser light. In Section IV, we conclude with a discussion of our results. 


\section{LASER BEAM DEFLECTION BY TRANSVERSE PLASMA FLOW}

Beam deflection has been a topic of vigorous interest in the past few years because of its potential to degrade the implosion symmetry of targets in gas-filled hohlraums. In 1982, Short, Bingham and Williams [12] analyzed the plasma dispersion relation with transverse flow, and showed that the spatial growth rate was enhanced and peaked at values of $M \ll$ 0 . A series of Nova gas-filled hohlraum experiments inspired H. A. Rose [13] to suggest that transverse plasma flow could explain the observed deflection of backscattered light. Hinkel, Williams and Still [14] simulated beam deflection for laser beams with spatial (RPP) and temporal (SSD [15]) beam smoothing. They showed that RPP beams deflect both supersonically (by forward Brillouin scatter between different $k$-components of the RPP beam) and subsonically as well, and that SSD mitigates the deflection. In this present work we extend our previous work by simulating unsmoothed beams, and by deriving an analytic expression, valid for circular and elliptic Gaussian beams for all values of the transverse flow Mach number, $M$. effects of beam deflection, and Ghosal and Rose [7] performed a linear analysis of beam deflection. Apart from the gas-filled hohlraum experiments on Nova, there have been exploding foil experiments performed on Nova [4] and on the Janus laser [5] at LLNL which show an intensity-dependent beam deflection.

The equations of motion governing the coupling of the laser light to the density perturbation [1] in the paraxial approximation are

$$
\begin{gathered}
\left(2 i k_{0} \partial_{z}+\nabla_{\perp}^{2}\right) \psi=\left(\omega_{p e}^{2} / c^{2}\right) \delta \bar{n} \psi \\
{\left[\left(\partial_{t}+u \partial_{x}+\nu\right)\left(\partial_{t}+u \partial_{x}\right)-C_{s}^{2} \nabla_{\perp}^{2}\right] \delta \bar{n}=C_{s}^{2}\left(v_{0} / v_{e}\right)^{2} \nabla_{\perp}^{2}|\psi|^{2}}
\end{gathered}
$$

Here, $\psi$ is the light wave vector potential scaled to its mean value, $\delta \bar{n}$ is the fractional density perturbation, $k_{0}$ is the laser wavenumber, $\omega_{p e}$ is the electron plasma frequency, $c$ is the speed of light, $\nu$ is the ion damping rate, $u$ is the transverse plasma flow, $C_{0}$ is the sound speed, $v_{0}$ is the electron quiver velocity in the light wave electric field, and $v_{e}$ is the electron 
thermal velocity. An additional approximation, consistent with the paraxial approximation, it that density perturbations are transverse to the forward-propagating light wave.

\section{A. Analysis}

A simple one-dimensional (1-D) argument demonstrates that the plasma density response is downstream from the light wave when transverse flow is present. Assume that the light wave is legislated, i.e., that $\psi$ is known and given. Then, in steady state, a 1-D version of Eqs. (1) takes the form

$$
\left[\left(u \partial_{x}+\nu\right) u \partial_{z}-C_{e}^{2} \partial_{x}^{2}\right] \delta \bar{n}=C_{e}^{2}\left(v_{0} / v_{e}\right)^{2} \partial_{x}^{2}|\psi|^{2}
$$

When $u=0$ (no transverse flow), then Eq. (2) can be integrated twice, yielding

$$
\delta \bar{n}=-\left(v_{0} / v_{e}\right)^{2}|\psi|^{2}
$$

i.e., pressure balance dictates that plasma density is expelled from regions of high intensity, and where the light wave has a local maximum, the plasma density has a local minimum.

If, however, $u, \nu \neq 0$, then Eq. (2) can be readily integrated once, giving,

$$
\left[\partial_{x}-\frac{u \nu}{C_{e}^{2}-u^{2}}\right] \delta \bar{n}=-\left(\frac{v_{0}}{v_{e}}\right)^{2} \partial_{x}|\psi|^{2} .
$$

A local density minimum means that $\partial_{x}(\delta \bar{n})=0$, and thus,

$$
\left.\delta \bar{n}\right|_{\partial_{x}(\delta \bar{n})=0}=\frac{C_{s}^{2}-u^{2}}{u \nu}\left(\frac{v_{0}}{v_{e}}\right)^{2} \partial_{x}|\psi|^{2},
$$

i.e., since $\nu, u>0$ and $\delta \bar{n}<0$ when evaluated at its minimum where $\partial_{x}(\delta \bar{n})=0$, it must be true that at this point, $\partial_{x}|\psi|^{2}<0$. Thus, the density well does not line up with the potential hump, rather, it is displaced downstream from the potential maximum where $\partial_{x}|\psi|^{2}<0$.

It now remains to show that the light wave is refracted into the displaced density well, thereby moving downstream as well. We obtain equations for the beam centroid and the beam width by taking intensity weighted moments of Eq. (1a), 


$$
\begin{gathered}
\frac{\partial^{2}}{\partial z^{2}}\left\langle\underline{x}_{\perp}\right\rangle=\frac{\partial}{\partial z}\left\langle\underline{\underline{k}}_{\perp}\right\rangle=-\frac{\omega_{p e}^{2}}{2\left(k_{0} c\right)^{2}}\left\langle\nabla_{\perp} \delta \bar{n}\right\rangle, \\
\frac{\partial^{2}}{\partial z^{2}}\left\langle\left(\underline{x}_{\perp}-\left\langle\underline{x}_{\perp}\right\rangle\right)^{2}\right\rangle=2\left\{\left\langle\left(\underline{\tilde{k}}_{\perp}-\left\langle\underline{\underline{k}}_{\perp}\right\rangle\right)^{2}\right\rangle-\frac{\omega_{p e}^{2}}{\left(k_{0} c\right)^{2}}\left\langle\left(\underline{x}_{\perp}-\left\langle\underline{x}_{\perp}\right\rangle\right) \cdot\left(\nabla_{\perp} \delta \bar{n}\right)\right\rangle\right\},
\end{gathered}
$$

where $\left\langle\underline{x}_{\perp}\right\rangle \equiv \int d^{2} x_{\perp} \psi^{*} \underline{x}_{\perp} \psi$, and $\left\langle\underline{\underline{k}}_{\perp}\right\rangle \equiv-\left(i / k_{0}\right) \int d^{2} x_{\perp} \psi^{*} \nabla_{\perp} \psi$, etc.

Eq. (6a) describes the beam centroid motion as a function of propagation distance. The transverse density gradient refracts the light to regions of lower electron density. Eq. (6b) governs the beam width as a function of propagation distance. As the beam propagates through plasma, the beam width will increase if diffraction [first term on right-hand side (RHS) of Eq. (6b)] dominates self-focussing [second term on RHS of Eq. (6b)], and it will decrease if self-focussing dominates diffraction. It is beyond the scope of this present work to self-consistently evolve the beam width. Heretofore we shall legislate a beam width and focus our attention on solving Eq. (6a).

We outline the methodology for solving Eq. (6a) by first solving for $\langle\delta \bar{n}\rangle$, where, again, the brackets denote an intensity-weighted average. By Fourier transforming Eq. (1b) and solving for $\delta \bar{n}\left(\underline{k}_{\perp}\right)$, we find that [14]

$$
\langle\delta \bar{n}\rangle=-\frac{1}{4 \pi^{2}}\left(\frac{v_{0}}{v_{e}}\right)^{2} \int \frac{d^{2} k_{\perp} k_{\perp}^{2}|\bar{\psi}|^{2}\left(\underline{k}_{\perp}\right)|\psi|^{2}\left(-\underline{k}_{\perp}\right)}{(1-M)^{2} k_{x}^{2}+k_{y}^{2}+i \bar{\nu} M k_{\perp} k_{x}}
$$

where $M \equiv u / C_{\imath}$ is the transverse Mach number, and $\bar{\nu} \equiv \nu /\left(k_{\perp} C_{s}\right)=$ constant is the ion damping decrement. Similarly, when the Fourier transform of Eq. (1b) is substituted into Eq. (6a), we obtain

$$
\frac{\partial}{\partial z}\left\langle\underline{k}_{\perp}\right\rangle=\frac{i \omega_{p e}^{2}}{2\left(k_{0} c\right)^{2}}\left(\frac{v_{0}}{v_{e}}\right)^{2} \int \frac{d^{2} k_{\perp} \underline{k}_{\perp} k_{\perp}^{2}|\tilde{\psi}|^{2}\left(\underline{k}_{\perp}\right)|\tilde{\psi}|^{2}\left(-\underline{k}_{\perp}\right)}{\left(1-M^{2}\right) k_{x}^{2}+k_{y}^{2}+i M \bar{\nu} k_{\perp} k_{x}} .
$$

Eqs. (7) and (8) can be integrated over angle for any wave field $|\tilde{\psi}|^{2}$ that can be expanded in a Fourier-Bessel series. However, for definiteness, we assume $|\tilde{\psi}|^{2}\left(\underline{k}_{\perp}\right)=$ $\exp \left[-k_{x}^{2} \sigma_{x}^{2} / 2-k_{y}^{2} \sigma_{y}^{2} / 2\right]$, i.e., $\psi\left(\underline{x}_{\perp}\right)$ is a Gaussian in real space with beam width $\sigma_{x}$ in the $x$-direction and $\sigma_{y}$ in the $y$-direction. 
It is then easy to perform the radial portion of the integrands of Eqs. (7) and (8), yielding

$$
\langle\delta \bar{n}\rangle=-\frac{1}{4 \pi^{2}}\left(\frac{v_{0}}{v_{e}}\right)^{2}\left\{1+\frac{M^{2}}{2 \pi} \frac{\sigma_{y}^{2}}{\sigma_{x}^{2}} \int_{0}^{2 \pi} \frac{d \theta \cos ^{2} \theta\left[\tilde{\alpha}^{2}-1-M^{2} \bar{\nu}^{2}\left(\sigma_{x}^{2} / \sigma_{y}^{2}\right) / 2-\epsilon^{2} \bar{\nu}^{2} \cos ^{2} \theta\right]}{\left[1-\left(1-\tilde{\alpha}^{2}\right) \cos ^{2} \theta\right]^{2}+\tilde{\beta}^{2} \cos ^{4} \theta}\right\},
$$

and

$$
\frac{\partial}{\partial z}\left\langle\underline{\underline{k}}_{x}\right\rangle=\frac{1}{32 \pi^{5 / 2}}\left(\frac{v_{0}}{v_{e}}\right)^{2} \frac{n}{n_{c}}\left(\frac{\omega_{0}}{k_{0} c}\right)^{2} \frac{M \bar{\nu} \sigma_{y}}{\sigma_{x}^{2}} \int_{0}^{2 \pi} \frac{d \theta \cos ^{2} \theta\left[1+\epsilon^{2} \cos ^{2} \theta\right]^{3 / 2}}{\left[1-\left(1-\tilde{\alpha}^{2}\right) \cos ^{2} \theta\right]^{2}+\tilde{\beta}^{2} \cos ^{4} \theta},
$$

where $\tilde{\alpha}^{2} \equiv\left(\sigma_{y}^{x} / \sigma_{x}^{2}\right)\left[1-M^{2}\left(1-\bar{\nu}^{2} / 2\right)\right]$, and $\tilde{\beta} \equiv\left(\sigma_{y}^{2} / \sigma_{x}^{2}\right)^{2} M^{2} \bar{\nu}$. The beam eccentricity is measured by $\epsilon^{2} \equiv \sigma_{y}^{2} / \sigma_{x}^{2}-1$. Note that in the above expression, the component of $\underline{\underline{k}}_{\perp}$ that is non-zero is that in the flow direction, i.e., $\left\langle\tilde{k}_{x}\right\rangle$.

If we use the identity $\cos ^{2} \theta=[1+\cos (2 \theta)] / 2$, make the change of variable $\phi=2 \theta$, and integrate about the unit circle by setting $z=\exp (i \phi)$, the expression for $\langle\delta \bar{n}\rangle$ can be determined by summing over the residues inside the unit circle,

$$
\begin{aligned}
\langle\delta \bar{n}\rangle=-\frac{1}{4 \tilde{\pi}^{2}}\left(\frac{v_{0}}{v_{e}}\right)^{2} & \left\{1+\frac{M^{2} \sigma_{\tilde{z}}^{2} / \sigma_{3}^{2}}{\sqrt{2}\left(\tilde{\alpha}^{2}+\tilde{\beta}^{2}\right)^{1 / 2}\left[\left(1-\tilde{\alpha}^{2}\right)^{2}+\tilde{\beta}^{2}\right]}\left\{\left[\tilde{\alpha}^{2}-1-M^{2} \bar{\nu}^{2}\left(\sigma_{y}^{2} / \sigma_{x}^{2}\right) / 2+\epsilon^{2} \bar{\nu}^{2}\right]\right.\right. \\
& \times\left\{\sqrt{2}\left(\tilde{\alpha}^{4}+\tilde{\beta}^{2}\right)^{1 / 2}-\left[\left(\tilde{\alpha}^{4}+\tilde{\beta}^{2}\right)^{1 / 2}+\tilde{\alpha}^{2}\right]^{1 / 2}\right\} \\
+ & {\left.\left.\left[\left(\tilde{\alpha}^{2}-1\right)\left[M^{2} \bar{\nu}^{2}\left(\sigma_{y}^{2} / \sigma_{x}^{2}\right) / 2-\epsilon^{2} \bar{\nu}^{2}\right]+\tilde{\beta}^{2}\right] \frac{\left[\left(\tilde{\alpha}^{4}+\tilde{\beta}^{2}\right)^{1 / 2}-\tilde{\alpha}^{2}\right]^{1 / 2}}{\tilde{\beta}}\right\}\right\} . }
\end{aligned}
$$

To determine the beam deflection rate, we perform the integration in Eq. (10) by expanding for small ellipticity, i.e., $|\epsilon|^{2} \ll 1$. Then along the unit circle, the second term in the square brackets of the numerator of Eq. (10) is small compared to the first, or $\left[1+\epsilon^{2} \cos ^{2} \theta\right]^{3 / 2} \simeq 1+(3 / 2) \epsilon^{2} \cos ^{2} \theta$. Summing over the residues, we then obtain

$$
\begin{aligned}
& \frac{\partial}{\partial_{z}}\left\langle\tilde{k}_{x}\right\rangle=\frac{\sqrt{2}}{32 x^{3 / 2}}\left(\frac{v_{0}}{v_{e}}\right)^{2} \frac{n}{n_{c}}\left(\frac{\omega_{0}}{k_{0} c}\right)^{2} \frac{M \bar{\nu}}{\sigma_{z}} \frac{\sigma_{y}}{\sigma_{x}}\left(\tilde{\alpha}^{4}+\tilde{\beta}^{2}\right)^{-1 / 2}\left\{\left[\left(\tilde{\alpha}^{4}+\tilde{\beta}^{2}\right)^{1 / 2}-\tilde{\alpha}^{2}\right]^{1 / 2} / \tilde{\beta}\right. \\
& +\frac{(3 / 2) \epsilon^{2}}{\left(1-\tilde{\alpha}^{2}\right)^{2}+\tilde{\beta}^{2}}\left[\sqrt{2}\left(\bar{\alpha}^{4}+\bar{\beta}^{2}\right)^{1 / 2}-\left[\left(\tilde{\alpha}^{4}+\tilde{\beta}^{2}\right)^{1 / 2}+\bar{\alpha}^{2}\right]^{1 / 2}\right. \\
& \left.\left.-\left[\left(\tilde{\alpha}^{2}-1\right) / \tilde{\beta}\right]\left[\left(\tilde{\alpha}^{4}+\tilde{\beta}^{2}\right)^{1 / 2}-\tilde{\alpha}^{2}\right]^{1 / 2}\right]\right\} \text {. }
\end{aligned}
$$


Fig. 1 depicts $\langle\delta \bar{n}\rangle$ vs. Mach number for both a circular beam (solid curve), where $\sigma_{y}=\sigma_{x}$, and for an elliptic beam (dashed curve), where $\sigma_{y}=2 \sigma_{x}$. In both cases, the density response near $M=1$ is almost triple the response at $M=0$. This demonstrates that the process of filamentation [14] is enhanced by near-sonic transverse flow.

The density response of the elliptic beam is slightly greater than that of the circular beam for $\epsilon^{2}>$ ) around the sonic surface. As $\sigma_{y}$ becomes large compared to $\sigma_{x}$, we recover the two-dimensional (2-D) limit, where, for $M>1$, the density response is positive, or there is a density enhancement rather than a density cavity.

In the subsonic regime, for a circular beam, where $1-M^{2} \gg M \bar{\nu}, M<1$, and $\epsilon=0$, the expression for $\delta \bar{n}$ as given by Eq. (11) reduces to

$$
\delta \bar{n}_{\text {subsonic }, \sigma_{x}=\sigma_{y}} \sim-\frac{1}{4 \pi^{2}}\left(\frac{v_{0}}{v_{e}}\right)^{2} \frac{1}{\sqrt{1-M^{2}}} .
$$

For near-sonic flow, we evaluate Eq. (11) with $M=1$ for a circular beam to find

$$
\delta \bar{n}_{\text {near-sonic, } \sigma_{x}=\sigma_{y}} \sim-\frac{1}{4 \pi^{2}}\left(\frac{v_{0}}{v_{e}}\right)^{2} \bar{\nu}^{-1 / 2}
$$

i.e., the density response for near-sonic flow is a resonant response that is limited by the damping, or, in the limit of weak damping, would be limited by nonlinear effects [16]. For supersonic transverse flow, where $M^{2}-1 \gg M \bar{\nu}$, we find that Eq. (11) reduces to

$$
\delta \bar{n}_{\text {supersonic }, \sigma_{x}=\sigma_{y}} \sim-\frac{\bar{\nu}}{8 \pi^{2}}\left(\frac{v_{0}}{v_{e}}\right)^{2} \frac{4+7 M^{2}-2 M^{4}}{\left(M^{2}-1\right)^{3 / 2}} .
$$

At $M=2$, the sign of $\delta \bar{n}$ changes, and the density response is an enhancement rather than a cavity.

In Fig. 2, we plot the beam deflection rate vs. Mach number for a circular beam (solid curve) and an elliptic beam (dashed curve). [In the circular beam limit, where $\sigma_{y}=\sigma_{x} \equiv \sigma$, only the first term in the large curly bracket survives of Eq. (12) survives.] The largest beam deflection rate occurs for near-sonic transverse flow. In the subsonic regime

$$
\frac{\partial}{\partial z}\left\langle\tilde{k}_{x}\right\rangle_{\text {subsonic }, \sigma_{x}=\sigma_{y}} \sim \frac{\sqrt{2}}{32 \pi^{3 / 2}}\left(\frac{v_{0}}{v_{e}}\right)^{2}\left(\frac{n}{n_{c}}\right)\left(\frac{\omega_{0}}{k_{0} c}\right)^{2} \frac{1}{\sigma} \frac{M \bar{\nu}}{\left(1-M^{2}\right)^{3 / 2}}
$$


Subsonically, the deflection rate scales linearly with the damping decrement and with Mach number, and increases as $M \rightarrow 1$.

In the near-sonic regime, for a circular beam, Eq. (12) reduces to

$$
\frac{\partial}{\partial z}\left\langle\tilde{k}_{x}\right\rangle_{\text {near-sonic }, \sigma_{x}=\sigma_{y}} \sim \frac{\sqrt{2}}{32 \pi^{3 / 2}}\left(\frac{v_{0}}{v_{e}}\right)^{2}\left(\frac{n}{n_{c}}\right)\left(\frac{\omega_{0}}{k_{0} c}\right)^{2} \frac{1}{\sigma \bar{\nu}^{1 / 2}}
$$

As with the density response, the beam deflection rate exhibits resonant behavior near $M=1$, which is limited by the inclusion of damping.

Finally, in the supersonic regime, Eq. (12) takes the form

$$
\frac{\partial}{\partial z}\left\langle\tilde{k}_{x}\right\rangle_{\text {supersonic }, \sigma_{x}=\sigma_{y}} \sim \frac{1}{16 \pi^{3 / 2}}\left(\frac{v_{0}}{v_{e}}\right)^{2}\left(\frac{n}{n_{c}}\right)\left(\frac{\omega_{0}}{k_{0} c}\right)^{2} \frac{1}{\sigma M} \frac{1}{\left(M^{2}-1\right)^{1 / 2}} .
$$

For $M \gg 1$, the beam deflection rate scales as $1 / M^{2}$, and is independent of the damping decrement. In all three regimes, the beam deflection rate scales with the figure of merit $Q \equiv\left(v_{0} / v_{e}\right)^{2}\left(n / n_{c}\right)\left(\omega_{0} / k_{0} c\right)^{2}$.

In these limiting regions, our beam deflection rate results are in agreement with those of Ghosal and Rose [7]. The pictorial results summarized in Figs. 1 and 2 indicate where we should concentrate our simulational efforts. Clearly, the largest response for both $\delta \bar{n}$ and the beam deflection rate is near $M=1$. In the weak damping limit, where a steady state is not reached, one would expect subsonic deflection from transient effects and self-induced bandwidth rather from damping, and near $M=1$, nonlinear effects would dominate the physics. In the experiments modelled in the next section, the plasma is composed of CH, with a damping decrement $\bar{\nu} \simeq 0.1$. In this regime we anticipate subsonic and supersonic deflection with the $M=1$ resonance limited by damping.

\section{B. Modelling Results}

As mentioned earlier in this article, gas-filled hohlraum experiments on the Nova laser [3] show a spot motion of $150 \mu \mathrm{m}$ toward the LEH that is reduced to $35 \mu \mathrm{m}$ when RPPs are used on the laser beams. Near peak laser power, the plasma density and transverse flow profiles 
as calculated by Lasnex [2] are as depicted in Fig. 3. The transverse flow profile is sheared, and is near sonic with a scale-length of about $600 \mu \mathrm{m}$ at the LEH. The corresponding plasma density is modelled as $n_{e} / n_{c}=.06+.04 \tanh \left(z / \lambda_{0}\right)$, where $\lambda_{0}=0.351 \mu \mathrm{m}$. Here, $z$ is chosen such that $n_{e} / n_{c}=0.1$ where $M=1$. The corresponding electron temperature is $T_{e}=3$ $\mathrm{keV}$.

It remains to specify the beam structure. The simulated unsmoothed Nova beam [6] $1 \mathrm{~mm}$ beyond best focus, which is the unsmoothed beam plane at the LEH, is markedly different in structure from the simulated RPP Nova beam [6] at best focus, which is its beam plane at the LEH. Both beams are $\mathrm{f} / 4$ and have similar average peak intensities. The speckle statistics of the beam are the same, but the unsmoothed Nova beam contains large coherent pieces, as well as large voids, which give rise to sub-spot-scale intensity modulations upon which the speckle statistics are superposed.

F3D models the speckle statistics of a beam given an average intensity about which the intensity varies. Computational limitations inhibit complete beam $(250 \mu \mathrm{m} \times 250 \mu \mathrm{m} \times$ $1000 \mu \mathrm{m}$ ) modelling in one F3D simulation. Thus, we have taken the unsmoothed and RPP beams, and averaged the intensities in the initial beam cross-section on a $28 \mu \mathrm{m} \times 28 \mu \mathrm{m}$ spatial scale, that is, the transverse dimension of a suite of 3-D F3D simulations.

These spatially smoothed average intensities are plotted in Fig. 4. The peak value for the RPP beam is $3 \times 10^{15} \mathrm{~W} / \mathrm{cm}^{2}$, as opposed to $1 \times 10^{16} \mathrm{~W} / \mathrm{cm}^{2}$ for the unsmoothed beam. An F3D simulation was performed at each spatially smoothed average intensity in the beam over a propagation distance of $350 \mu \mathrm{m}$. At $z=350 \mu \mathrm{m}$, each piece of the beam is placed back in its original beam position, and the entire beam is paraxially propagated through 1 $\mathrm{mm}$ of plasma at density $n=0.1 n_{c}$, roughly the distance from the LEH to the wall. The intensity distribution vs. wall position of the RPP and unsmoothed beams is plotted in Fig. 5. The intensity-weighted centroid of the RPP beam is located $40 \mu \mathrm{m}$ from where the beam was pointed, whereas the centroid of the unsmoothed beam is located $110 \mu \mathrm{m}$ closer to the LEH than it was pointed, in good agreement with experimental results. Thus we see that it is the difference in the intensity distributions of the beams, i.e., the beam structure, that 
accounts for the change in wall position for unsmoothed vs. RPP beams.

A secondary feature that also enhances the deflection of the unsmoothed beam arises from the focusing geometry. The unsmoothed $\mathrm{f} / 4$ Nova beam is focused $1 \mathrm{~mm}$ outside the $\mathrm{LEH}$, and in the beam plane at the LEH, the hot spots in the beam are more accurately described by $\mathrm{f} / 6$ speckle statistics. Since an $\mathrm{f} / 6$ speckle is longer [by a factor of $(3 / 2)^{2}$ ] and wider [by a factor of $3 / 2$ ] than a $f / 4$ speckle, the spatial region of a given hot spot over which beam deflection can occur is larger, thus yielding more beam deflection.

\section{CHANNEL FORMATION BY ULTRA-INTENSE LASER LIGHT}

Channeling of intense laser light is crucial to the fast ignitor concept [11], where light must reach the overdense regime. Prior to channeling the intense laser light, a channel must be created by the "hole-boring" pulse. We have begun to examine and analyze beam propagation in underdense plasma in an effort to better understand the channel formation process.

We utilize a relativistic form of F3D [17] to study channeling. In this form, the index of refraction in the light wave equation has been modified according to the replacement $n_{e} \rightarrow n_{e} / \gamma$, where $\gamma \equiv\left(1+u_{0}^{2} / c^{2}\right)^{1 / 2}$, with $u_{0} \equiv e A_{0} /\left(m_{e} c\right)$. [Here, $A_{0}$ is the vector potential of the light wave.] This accounts for relativistic corrections to the electron mass. We modified the ponderomotive drive in the momentum equation by making the replacement $\nabla u_{0}^{2} \rightarrow 2 c^{2} \nabla \gamma$, so that momentum is conserved. At very high intensities, the ponderomotive drive then scales as $\nabla u_{0}$ rather than as $\nabla u_{0}^{2}$ as it does at moderate intensities where $\gamma \approx 1$. Finally, we have implemented a charge separation model similar to that of Sun et al. [18], where $n_{e}=\max \left[0, Z n_{i}+\left(m_{e} c^{2}\right) /\left(4 \pi e^{2}\right) \nabla_{\perp}^{2} \gamma\right]$. Here, $n_{i}$ is the ionic density, $Z$ is the ionic charge, $m_{e}$ is the electronic mass, and $e$ is the electronic charge. This charge separation model permits the electrons to be blown out of the channel by the ponderomotive force on very short (sub-picosecond) time scales, inducing a charge separation which then draws the ions out of the channel as well. 
We have propagated a 30 ps FWHM (full width at half max) Gaussian beam with peak intensity $5.7 \times 10^{17} \mathrm{~W} / \mathrm{cm}^{2}$ and a waist of $15 \mu \mathrm{m}$ at best focus through $1 \mathrm{~mm}$ of underdense plasma at density $n_{e}=0.1 n_{c}$ and electron temperature $T_{e}=2 \mathrm{keV}$. The beam filaments into several pieces, and the main (central filament) contains $65-70 \%$ of the intensity. The laser beam has self-focussed by an order of magnitude, because of both relativistic and ponderomotive effects. The central filament plasma density is at about $n_{e}=0.01 n_{c}$, and a channel forms through the entire $1 \mathrm{~mm}$ of plasma. We find this process occurs for a range of FWHM spot sizes from $3-15 \mu \mathrm{m}$.

We have also simulated beams with both random phase aberrations and spherical aberrations. Figs. 6 show the $x=0$ slice of the laser amplitude (Fig. 6a) and the plasma density (Fig. $6 \mathrm{~b}$ ) for an $\mathrm{f} / 3$ laser beam with random phase aberrations which expand the spot size at best focus to $15 \mu \mathrm{m}$. In Fig. $6 \mathrm{a}$ we observe 3 hot spots near best focus, located at $z=180 \mu \mathrm{m}$, which have self-focussed and thereby increased the intensity by a factor of 2. In Fig. 6b, plasma has been pushed out of the regions where the beam is most intense. The beam structure, i.e., the presence of multiple hot spots, locally increases the intensity, but not globally. Thus relativistic and ponderomotive self-focussing is limited to the hot spot regions, from which plasma density is expelled. With either random phase or spherical aberrations, a channel does not form through $400 \mu \mathrm{m}$ of $n_{e}=0.1 n_{c}$ plasma in $30 \mathrm{ps}$. We thus conclude that aberrated beams do not channel as effectively as diffraction limited beams.

\section{CONCLUSIONS}

In summary, we have shown that a predictive capability requires a model that contains the important beam structure characteristics. We have presented two examples where the beam structure was essential.

In the first example, we studied beam deflection caused by transverse flow. We derived a global formula for the density response and beam deflection rate, valid for all Mach numbers provided the damping is not weak. In the weak damping limit, where a steady state is 
not reached, the damping decrement $\bar{\nu}$ can be replaced with the magnitude of the transient density response, $\partial_{t} \delta \bar{n} / \delta \bar{n}$. In practice, the $\nu \rightarrow 0$ limit is never achieved because of residual time dependent effects such as transients, self-induced bandwidth, and background evolution. Also, in the weak damping limit, nonlinear effects become important in the near-sonic regime. In the subsonic regime, the beam deflèction rate scales linearly with the damping decrement and with Mach number, and scales inversely with $\left(1-M^{2}\right)^{3 / 2}$. The density response is independent of the damping decrement in this regime, and scales inversely as $\left(1-M^{2}\right)^{1 / 2}$. Around $M=1$, damping limits the resonant response, and both the beam deflection rate and the density response scale as $\bar{\nu}^{-1 / 2}$. In the supersonic regime, the beam deflection rate is independent of the damping decrement, and scales as $1 /\left[M\left(M^{2}-1\right)^{1 / 2}\right]$. Here, the density response is proportional to the damping decrement, and scales with Mach number such that at $M=2$, there is a transition from a density cavity to a density enhancement. It also scales as $1 /\left(M^{2}-1\right)^{3 / 2}$. Such analysis can be used to formulate a reduced description in models where laser propagation is not treated in detail [2].

We have used this information to focus our modelling efforts where the transverse flow is near-sonic, which, in gas-filled hohlraums, is around the LEH. When the marked difference in the intensity distributions of the unsmoothed and RPP beams is folded into our modelling, we find that the intense portions of the unsmoothed beam deflect more (in agreement with our figure-of-merit scaling), thereby moving the wall spot centroid of the unsmoothed beam closer to the LEH than that of the RPP beam. Our results agree quantitatively with the $\mathbf{x}$-ray emission data in gas-filled hohlraum experiments [3].

In our second example, we studied the effect of beam structure on channeling of intense laser beams. We determined that an idealized beam, containing only one hot spot with a peak intensity at $5.7 \times 10^{17}$, successfully creates a channel through underdense plasma at density $n_{e}=0.1 n_{c}$. If, however, a more realistic beam model, such as one containing random phase aberrations, but with the same average power as that of the idealized beam, is used, we find that a channel does not form. Plasma density is expelled from the regions where the beam has hot spots, but builds up between the hot spots. This work has set in place the 
required framework to perform further analysis, such as determining the intensity threshold as a function of beam structure above which channel formation occurs.

\section{ACKNOWLEDGEMENTS}

We acknowledge valuable input from H. A. Rose, S. G. Glendinning, L. J. Suter, B. F. Lasinski, K. G. Estabrook, and M. H. Key. This work was performed under the auspices of the U.S. Department of Energy by the Lawrence Livermore National Laboratory under Contract No. W-7405-ENG-48. 


\section{REFERENCES}

[1] J. F. Drake, P. K. Kaw, Y. C. Lee, G. Schmidt, C. S. Liu, and M. N. Rosenbluth, Phys. Fluids 17, 778 (1974); D. W. Forslund, J. M. Kindel, and E. L. Lindman, Phys. Fluids 18, 1002 (1975); A. Simon and W. B. Thompson, editors, Advances in Plasma Physics, Vol. 6 (John Wiley and Sons, Inc., New York, NY, 1976).

[2] G. Zimmerman and W. L. Kruer, Comments Plasma Phys. Control. Fusion 2, 85 (1975).

[3] S. G. Glendinning and L. V. Powers, private communication, 1996; N. Delamater, T. Murphy, A. Hauer, R. Kauffman, A. Richard, E. Lindman, G. Magelssen, B. Wilde, D. Harris, B. Failor, J. Wallace, L. Powers, S. Pollaine, L. Suter, R. Chrien, T. Shepard, H. Rose, E. Williams, M. Nelson, M. Cable, J. Moore, M. Salazar, and K. Gifford, Phys. Plasmas 3, 2022 (1996).

[4] J. D. Moody, B. J. MacGowan, D. E. Hinkel, W. L. Kruer, E. A. Williams, K. Estabrook, R. L. Berger, R. K. Kirkwood, D. S. Montgomery, and T. D. Shepard, Phys. Rev. Lett, 77, 1294 (1996).

[5] P. E. Young, D. E. Hinkel, C. H. Still, R. L. Berger, K. G. Estabrook, J. H. Hammer, W. L. Kruer, and E. A. Williams, Bull. Am. Phys. Soc. 41, 1599 (1996).

[6] R. A. Sacks, M. A. Henesian, S. W. Haney, J. B. Trenholme, ICF Annual Report, Lawrence Livermore National Laboratory, UCRL-LR-105821-96, 207 (1996).

[7] S. Ghosal and H. A. Rose, Phys. Plasmas 4, 2376 (1997).

[8] R. L. Berger, B. F. Lasinski, T. B. Kaiser, E. A. Williams, A. B. Langdon, and B. I. Cohen, Phys. Fluids B 5, 2243 (1993).

[9] P. Wegner, Report No. UCRL-ID-110480, Vol. 2, 1992.

[10] Y. Kato and K. Mima, Appl. Phys. B 29, 186 (1982). 
[11] M. Tabak, J. Hammer, M. E. Glinsky, W. L. Kruer, S. C. Wilks, J. Woodworth, E. M. Campbell, M. D. Perry, and R. J. Mason, Phys. Plasmas 1, 1626 (1994).

[12] R. W. Short, R. Bingham, and E. A. Williams, Phys. Fluids 25, 2302 (1982).

[13] H. A. Rose, private communication, LLNL, 1995.

[14] D. E. Hinkel, E. A. Williams, and C. H. Still, Phys. Rev. Lett. 77, 1298 (1996).

[15] S. Skupsky, R. W. Short, T. Kessler, R. S. Craxton, S. Letzring, and J. M. Soures, J. Appl. Phys. 66, 3456 (1989).

[16] W. L. Kruer and J. H. Hammer, Comments Plasma Phys. Control. Fusiion 18, 85 (1997).

[17] D. E. Hinkel, A. B. Langdon, and C. H. Still, "Channeling and Filamentation of Intense Laser Light in Underdense Plasma", presented at the 27th annual Anomalous Absorption Conference, June 1-5, 1997, Vancouver, British Columbia, Canada.

[18] G.-Z. Sun, E. Ott, Y. C. Lee, and P. Guzdar, Phys. Fluids 30, 526 (1987); M. D. Feit, J. C. Garrison, and A. M. Rubenchik, Phys. Rev. E 56, 2394 (1997). 


\section{FIGURES}

FIG. 1. The average fractional density perturbation, $\delta \bar{n}$, normalized to $4 \pi^{2}\left(v_{e} / v_{0}\right)^{2}$, vs. Mach number when the ion damping decrement $\bar{\nu}=0.1$. The solid curve is for a circular beam, and the dashed curve is for an elliptic beam, where $\sigma_{y}^{2} / \sigma_{x}^{2}=2$.. The average value of $\delta \bar{n}$ is 2.5 times greater for the circular beam in near-sonic transverse flow than in the absence of transverse flow.

FIG. 2. Beam deflection rate vs. Mach number when the ion damping decrement $\bar{\nu}=0.1$. The solid curve is for a circular beam, and the dashed curve is for a slightly elliptical beam, where $\sigma_{y}^{2} / \sigma_{x}^{2}=1.1$. The primary contribution to the beam deflection rate occurs when the transverse flow is nearly sonic.

FIG. 3. The transverse flow and plasma density $\left(n_{e} / n_{c}\right)$ profiles along a typcial ray path in a methane-filled hohlraum near peak power on the Nova laser as calculated by Lasnex. The corresponding electron temperature is $T_{e} \sim 3 \mathrm{keV}$, and the laser light wavelength is $\lambda_{0}=0.351 \mu \mathrm{m}$.

FIG. 4. The running sum of the intensity distributions of RPP and unsmoothed Nova beams vs. intensity when the intensity is spatially averaged on a $28 \mu \mathrm{m} \times 28 \mu \mathrm{m}$ scale. The RPP beam has a peak spatially averaged intensity of $3 \times 10^{15} \mathrm{~W} / \mathrm{cm}^{2}$, whereas the unsmoothed beam peaks at $1 \times 10^{16} \mathrm{~W} / \mathrm{cm}^{2}$.

FIG. 5. The intensity distribution vs. wall position of the RPP and unsmoothed Nova beams near peak power. The centroid of the RPP beam is located at $40 \mu \mathrm{m}$, whereas that of the unsmoothed beam is located at $110 \mu \mathrm{m}$.

FIG. 6. The laser amplitude and plasma density for a phase aberrated $\mathrm{f} / 3$ beam with a spot size of $15 \mu \mathrm{m}$ at best focus and $40 \mathrm{TW}$ of input power. After $15 \mathrm{ps}$, the beam has failed to channel through $400 \mu \mathrm{m}$ of plasma at density $n_{e}=0.1 n_{c}$, whereas a diffraction limited $\mathrm{f} / 3$ beam creates an evacuated channel within 15 ps. 


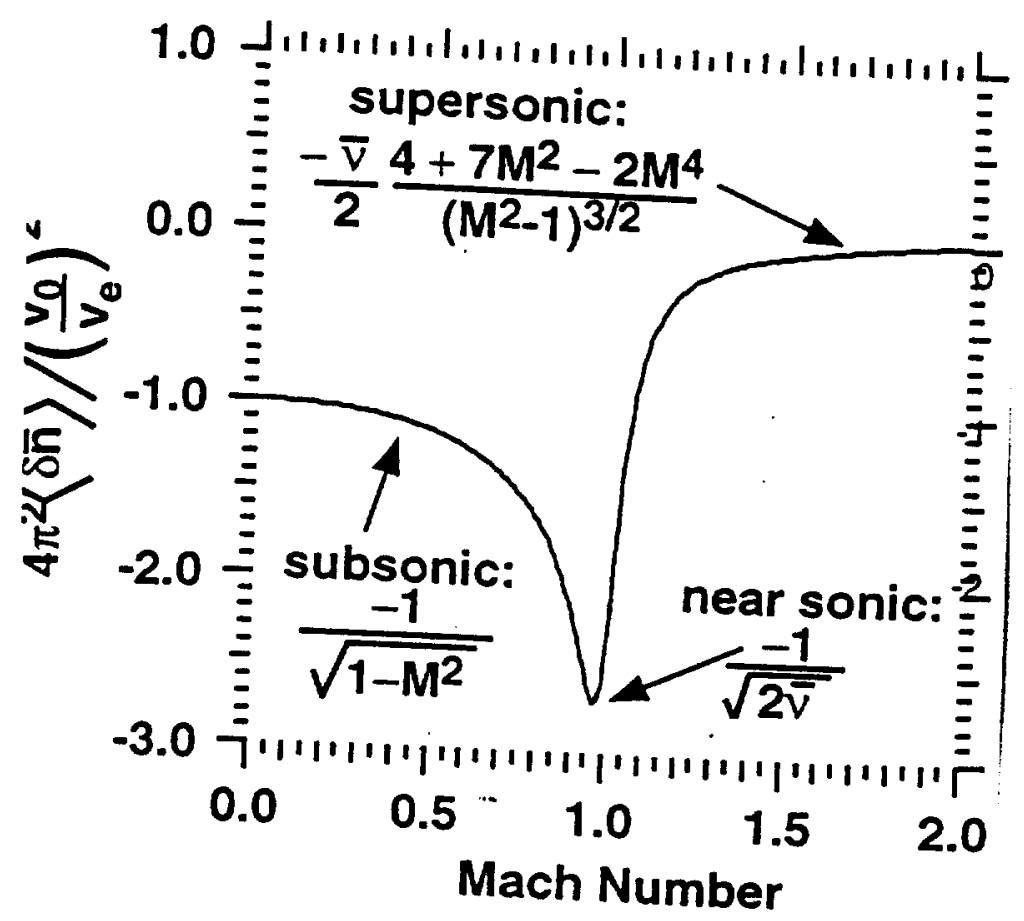

Figure 1 


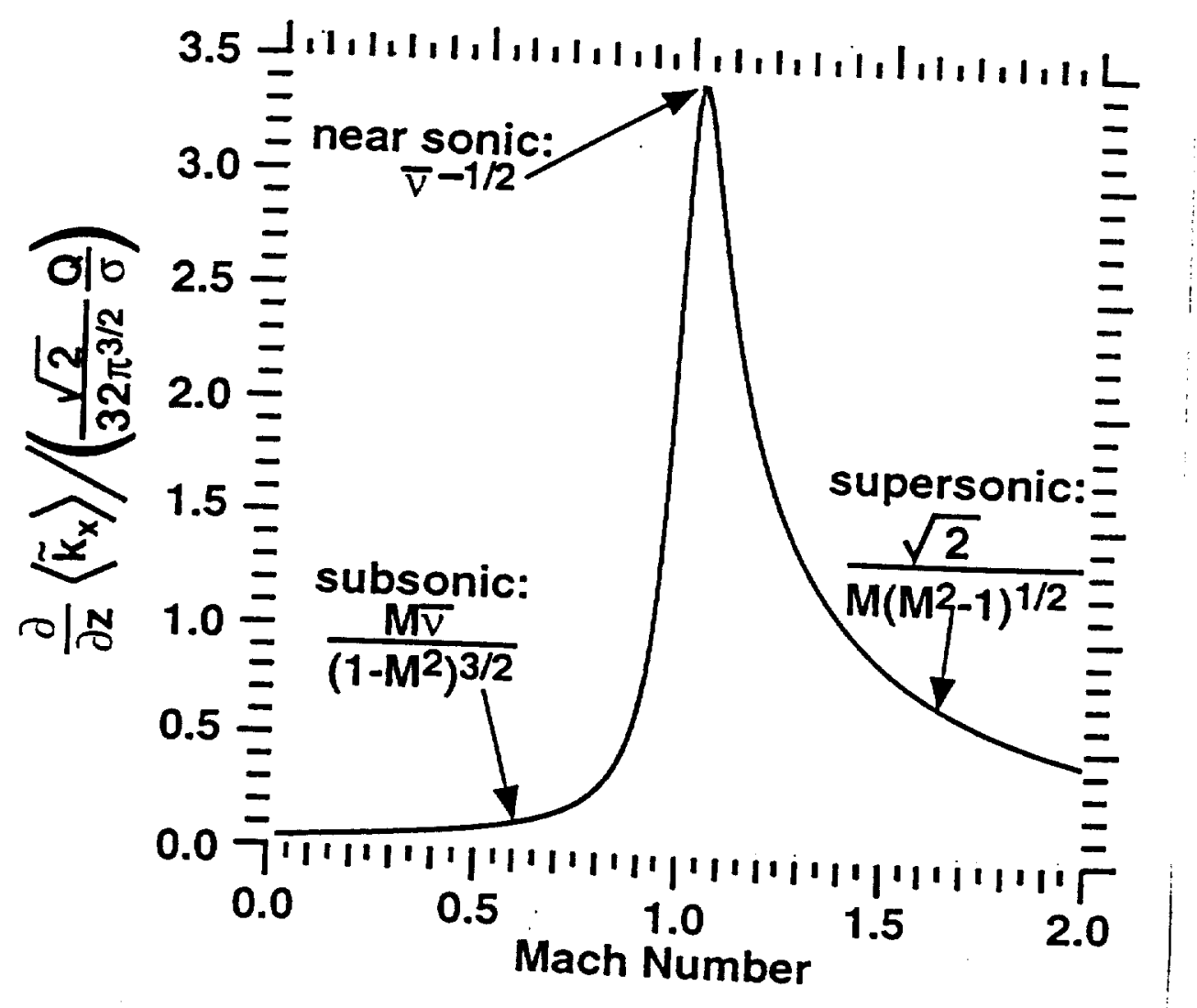

Figure 2 


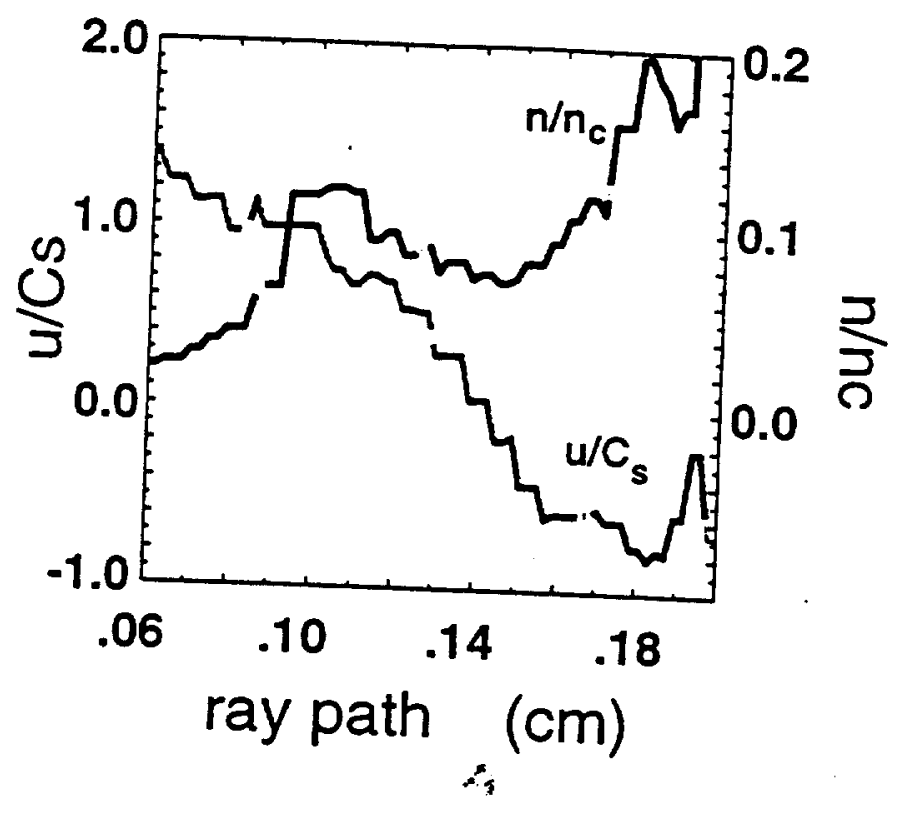

Figure 3 


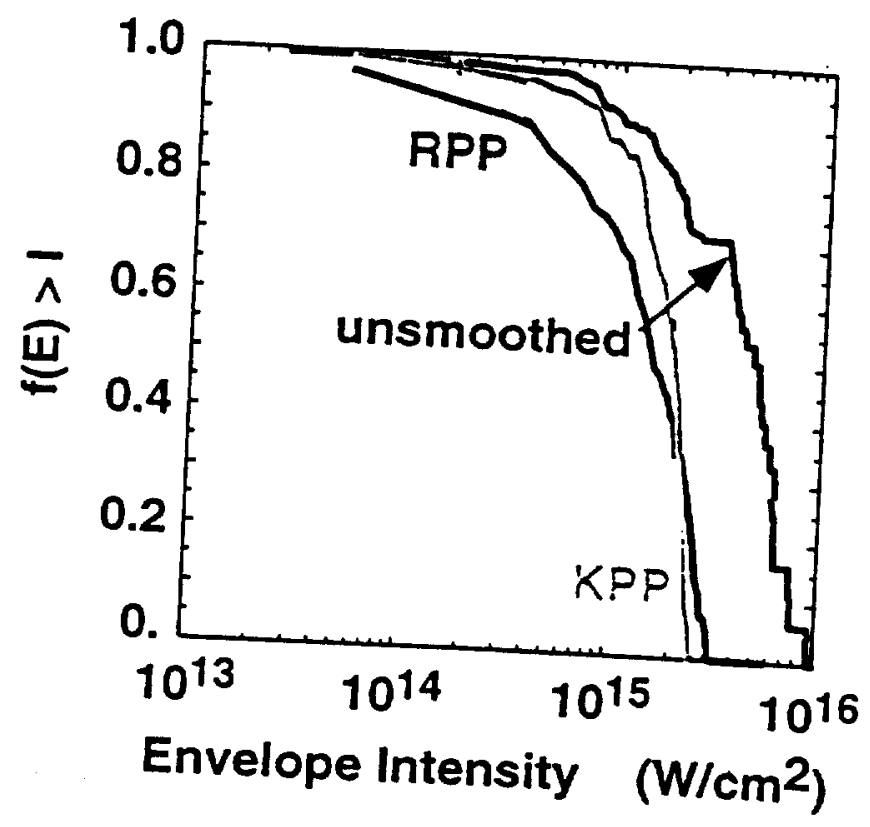

Figure 4 


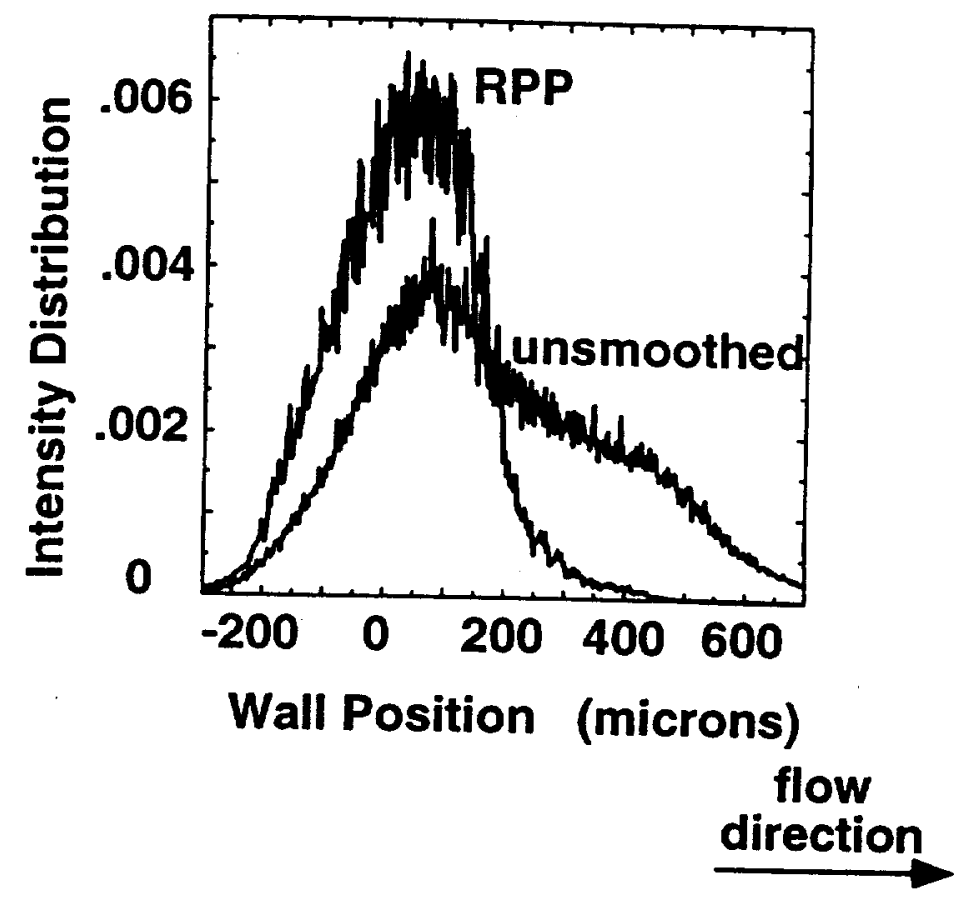

Figure 5 


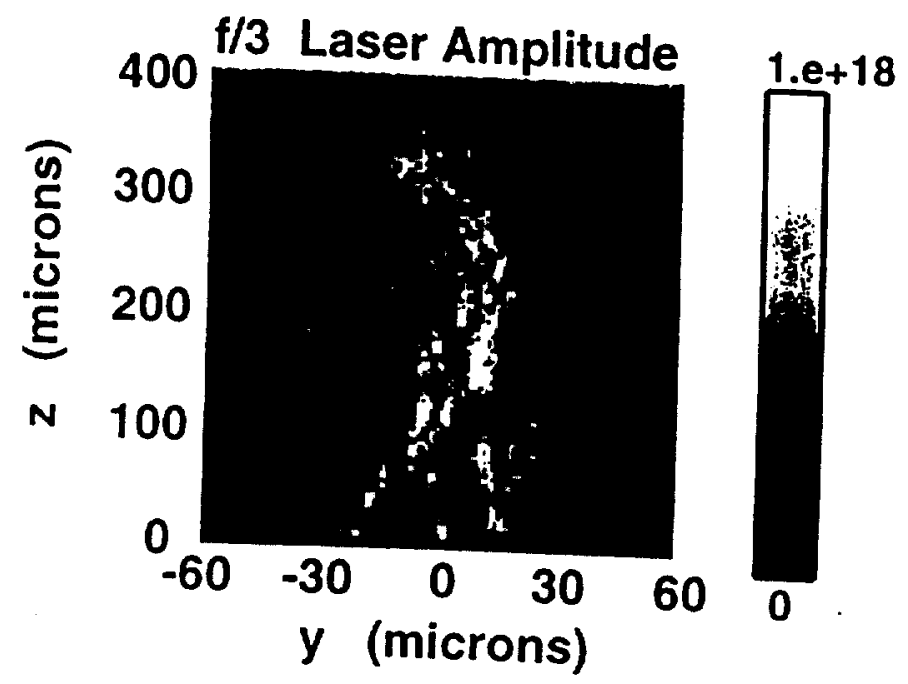

Figure $6 a$

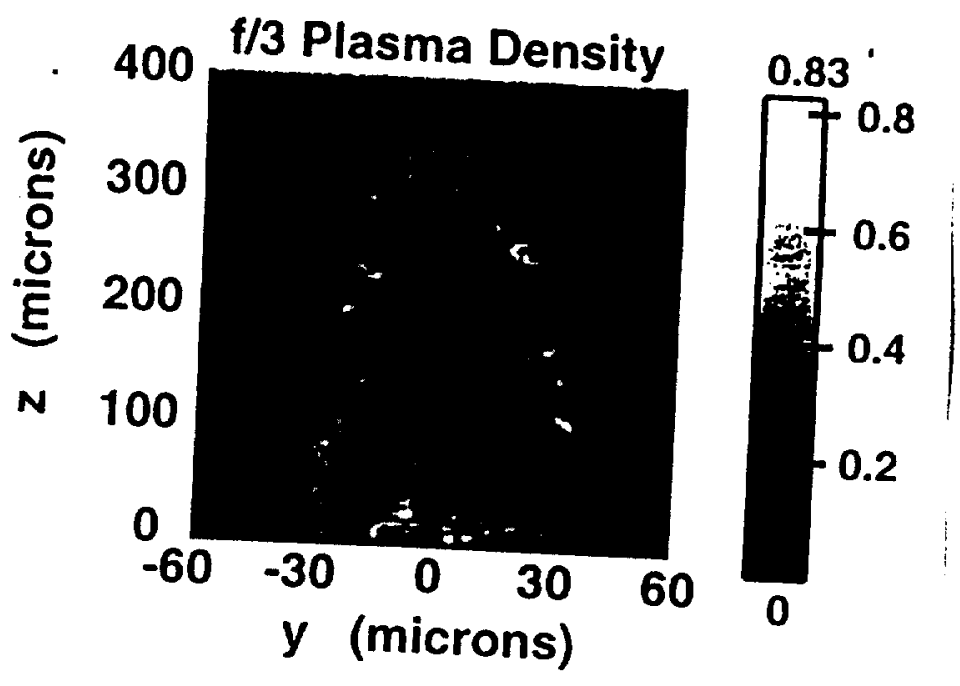

Figure $6 b$

Figures 6 


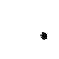




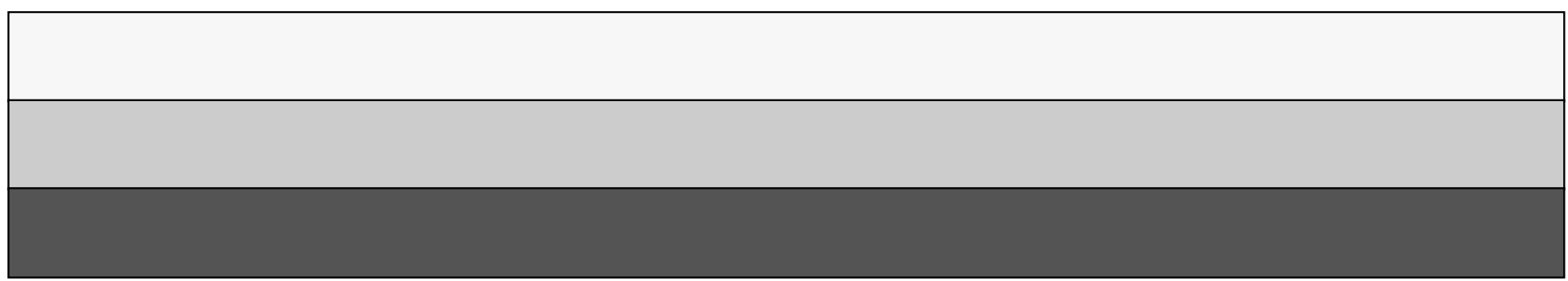

